\title{
Controlled Aspiration and Positioning of Biological Cells in a Micropipette
}

\author{
XuPing Zhang, Member, IEEE, Clement Leung, Zhe Lu, Member, IEEE, Navid Esfandiari, \\ Robert F. Casper, and Yu Sun*, Senior Member, IEEE
}

\begin{abstract}
Manipulating single cells with a micropipette is the oldest, yet still a widely used technique. This paper discusses the aspiration of a single cell into a micropipette and positioning the cell accurately to a target position inside the micropipette. Due to the small volume of a single cell (picoliter) and nonlinear dynamics involved, these tasks have high skill requirements and are labor intensive in manual operation that is solely based on trial and error and has high failure rates. We present automated techniques in this paper for achieving these tasks via computer vision microscopy and closed-loop motion control. Computer vision algorithms were developed to detect and track a single cell outside and inside a micropipette for automated single-cell aspiration. A closed-loop robust controller integrating the dynamics of cell motion was designed to accurately and efficiently position the cell to a target position inside the micropipette. The system achieved high success rates of $98 \%$ for cell detection and $97 \%$ for cell tracking $(n=100)$. The automated system also demonstrated its capability of aspirating a single cell into a micropipette within $2 \mathrm{~s}$ (versus 10 $s$ by highly skilled operators) and accurately positioning the cell inside the micropipette within $8 \mathrm{~s}$ (versus $25 \mathrm{~s}$ by highly skilled operators).
\end{abstract}

Index Terms-Aspiration, automation, cell manipulation, cell transfer, computer vision microscopy, dynamic modeling, micropipette, robust control, visual servo.

\section{INTRODUCTION}

$\mathbf{S}$ INGLE-CELL manipulation techniques have been under intense development with the advent of many single-cell analysis approaches [1]-[3]. For single-cell analysis, a cell must be positioned at defined locations for testing [2], [3]. Foreign materials and cellular structures need to be inserted [4] into or extracted [5] from single cells for testing cellular response to molecules or for genetic manipulation/diagnostics.

Among existing cell manipulation techniques, including microfluidics-based positioning [6], magnetic localization [7], optical trapping [8], dielectrophoresis [9], acoustics-based ma-

Manuscript received October 28, 2011; accepted December 21, 2011. Date of publication January 3, 2012; date of current version March 21, 2012. This work was supported by the Natural Sciences and Engineering Research Council of Canada and by the Canada Research Chairs Program.X. P. Zhang, C. Leung, and Z. Lu Shared first authorship. Asterisk indicates corresponding author.

X. P. Zhang, C. Leung, and Z. Lu are with the Advanced Micro and Nanosystems Laboratory, University of Toronto, Toronto, ON M5S 3G8, Canada (e-mail: zhxp@mie.utoronto.ca; clement.leung@utoronto.ca; zhe.lu@utoronto.ca).

N. Esfandiari and R. F. Casper are with the Department of Obstetrics and Gynaecology, University of Toronto, Toronto, ON M5S 3G8, Canada (e-mail: nesfand@yahoo.com; casper@lunenfeld.ca).

*Y. Sun is with the Advanced Micro and Nanosystems Laboratory, University of Toronto, Toronto, ON M5S 3G8, Canada (e-mail: sun@mie.utoronto.ca).

Color versions of one or more of the figures in this paper are available online at http://ieeexplore.iee.org.

Digital Object Identifier 10.1109/TBME.2012.2182673 nipulation [10], [11], and micropipette manipulation [12], the micropipette technique has the longest history and is still one of the most widely used techniques, particularly in oocyte and embryo manipulation (e.g., injection and retrieval of cellular materials) [13], [14], and for single-cell pick-place and transfer [15], [16], due to the high specificity and high precision the technique offers.

The task discussed in this paper is the aspiration of a single cell into a micropipette and positioning the cell inside the micropipette, in a highly controlled, automated manner. This task is relevant to a number of biological and clinical applications. For instance, when transferring a single cell from one droplet of culture medium to a defined location outside the droplet, aspirating the cell into a micropipette is important to avoid exposing the cell to the air environment. In clinical intracytoplasmic sperm injection (ICSI) [17], [18], a single sperm cell must be aspirated into a micropipette and accurately positioned inside the micropipette before the sperm is inserted into an oocyte for in vitro fertilization.

Aspirating a single cell into a micropipette and precisely controlling the cell's position within the micropipette is challenging, due to the small volume of a cell (picoliter) and the nonlinear dynamics involved in the process (e.g., varying mass of culture medium entering the micropipette in cell aspiration). In manual operation, an operator looks through the eyepieces of a microscope and operates multiple devices (microscope stage, micromanipulator, pump, etc.). When the micropipette approaches a target cell, a small negative pressure is applied to aspirate the cell into the micropipette. Once the cell enters the micropipette, which is a rapid event, the operator must quickly apply a positive pressure to stop the cell movement so that the cell does not enter too far into the micropipette and disappears. In order to position the cell to a desired location inside the micropipette, the operator must repeatedly adjust the application of negative and positive pressure skillfully.

High skill requirements and labor intensiveness in manual aspiration of single cells call for automation and the reduction of human involvement. Automated cell aspiration and positioning requires visual tracking of single cells and the development of a controller for precision cell position control. Significant progress has been made in computer vision microscopy for cell detection and tracking [19]-[24]; however, automated detection and tracking of a single cell inside a micropipette, where contaminants/particles can easily occlude a single cell, has not been explored. In addition, positioning a cell inside the micropipette involves nonlinear dynamics. The mass of culture medium containing the cell changes with time, and system damping varies 
with cell culture medium viscosity. The nonlinear dynamics requires appropriate controller design for precisely and stably moving a cell to a target position inside the micropipette.

This paper presents automation techniques (dynamic modeling and vision-based robust control) for automated single-cell aspiration into a micropipette and positioning the cell precisely inside the micropipette. Cell detection and visual tracking algorithms were developed. A nonlinear dynamic model was established to describe cell motion inside the micropipette. With considerations of the nonlinearity and variation of mass and damping, a robust controller was developed to move a cell to a target position.

The techniques presented in this paper have general applicability to the aspiration and positioning of all suspended cells. Human sperm cells were used in experimentation for evaluating the performance of the algorithms and controller. Based on experiments on over 100 sperm cells, the system demonstrated its capability for automated cell aspiration, and the vision-based robust controller is capable of efficiently and accurately moving a cell to a target position inside the micropipette.

\section{METHODS}

Human sperm cells were used in this study. Sperm manipulation is a required step in ICSI where a single sperm cell must be tracked, immobilized, and aspirated into a sharp injection micropipette, before it is injected into an oocyte. To minimize the amount of sperm culture medium injected into the oocyte for preserving the oocyte's development competence, the tail of the sperm cell inside the micropipette must be positioned close to the micropipette tip. We previously reported methods for tracking motile sperm cells and robotically immobilizing them [24]. This paper presents automated aspiration and positioning of immobilized sperm cells.

In the system, a linear stage (Newmark Systems, ET-100-10, travel range: $100 \mathrm{~mm}$, resolution: $0.04 \mu \mathrm{m}$ ) was used to drive a $250-\mu \mathrm{L}$ glass syringe (Hamilton). Volume control resolution is $0.1 \mathrm{pL}$. The syringe was filled with mineral oil and connected to a straight ICSI micropipette (MIC-50-0, Humagen) through a connecting tube and a micropipette holder. The micropipette holder was mounted on a 3-DOF motorized micromanipulator (MP285, Sutter Inc.). An motorized $X Y$ stage (Prior Scientific, H117, travel range: $114 \mathrm{~mm} \times 76 \mathrm{~mm}$, resolution: $0.01 \mu \mathrm{m}$ ) was used to hold and position a petri dish containing culture medium and sperm cells. Cell manipulation was conducted under a standard inverted microscope (Nikon Ti-S) connected to a CMOS camera (601f, Basler). The camera provided visual feedback at $30 \mathrm{~Hz}$ and communicated with a host computer that controls all devices and processes images.

Fig. 1 illustrates the operation flow of automated sperm aspiration. The micropipette is first positioned close to the immobilized sperm cell [see Fig. 1(a)]. A negative pressure is then applied to aspirate the sperm cell into the micropipette. The system stops the application of low negative pressure once detecting the sperm cell entering the micropipette. For positioning the sperm cell inside the micropipette, the cell is visually tracked
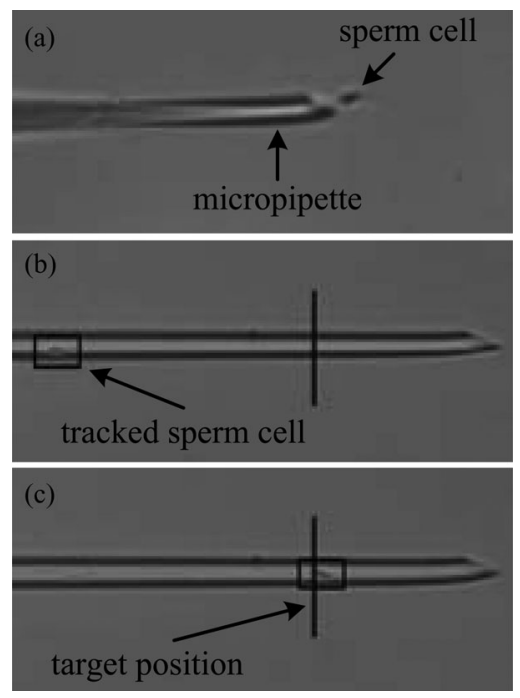

Fig. 1. Operation flow of automated sperm aspiration. (a) Align the micropipette to immobilized sperm cell. (b) Visually track the sperm cell. (c) Position the sperm cell to target position.

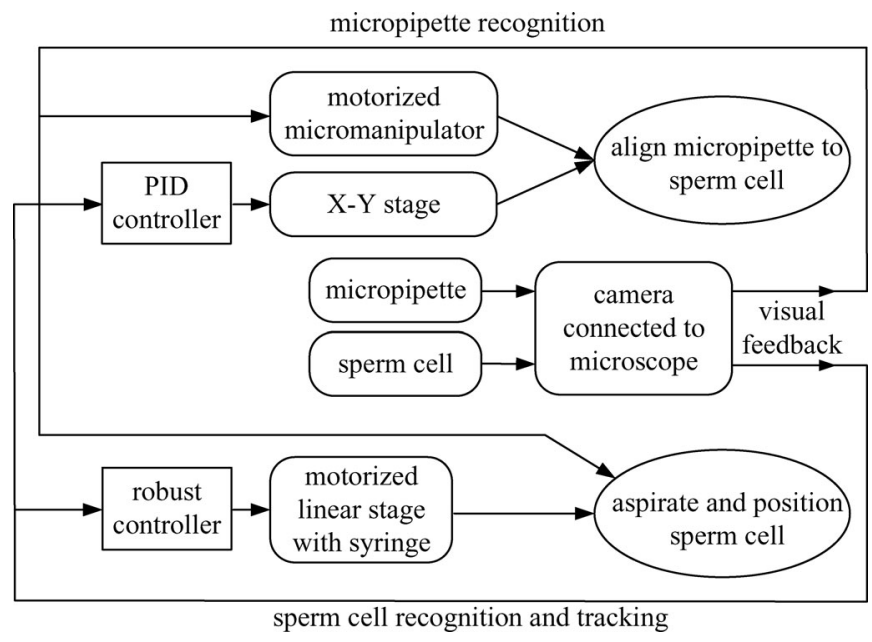

Fig. 2. Control architecture of the system.

[see Fig. 1(b)], and a controller moves the sperm cell to the target position [vertical line shown in Fig. 1(c)].

The overall control architecture of the system is described in Fig. 2. Microscopy visual feedback is a critical modality used for recognizing the micropipette and sperm cell. Furthermore, visual tracking is used for providing positing feedback to guide the operation of the micromanipulator, the $X Y$ translational stage, and the motorized linear stage with a syringe. A robust controller controls the motion of the linear stage for accurately and quickly positioning the sperm cell to a target position inside the micropipette.

\section{Visual Detection AND TRACKING}

Automated aspiration and positioning requires visual detection of the sperm cell at the micropipette entrance and visual tracking of the sperm cell inside the micropipette. 


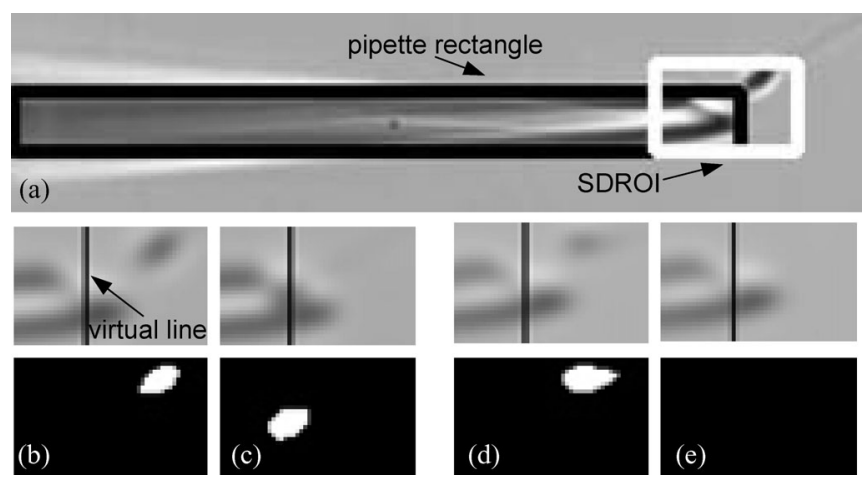

Fig. 3. Detecting sperm cell's entrance into micropipette. (a) SDROI is represented by the white box. The fitted micropipette rectangle is shown in black. (b) and (c) Sperm passed the virtual line. (d) and (e) Sperm was detected to the right of the virtual line. In (e), the sperm disappeared from the SDROI.

\section{A. Sperm Detection}

To prepare for aspiration, the system positions the micropipette tip $5 \mu \mathrm{m}$ (along both $X$ and $Y$ ) from the immobilized sperm cell [see Fig. 3(a)]. The detection algorithm starts by finding the region of the image that contains the micropipette tip and the sperm cell to be aspirated. This region labeled as the sperm detection region of interest (SDROI) is where image processing is performed to detect the sperm cell entering the micropipette [see Fig. 3(a)]. To find the SDROI, an identification algorithm locates the micropipette tip in the first frame of image. The SDROI is then extrapolated from the micropipette tip position.

The algorithm applies Otsu adaptive thresholding to the original image to create a binary image, which separates the foreground from the background of the original image. Contour detection is then applied to the binary image. The micropipette is recognized by finding the contour with the largest area. An upright bounding rectangle (the pipette rectangle) is fitted to the contour. The right side of the pipette rectangle gives the position of the micropipette tip. Using the coordinates of the pipette rectangle, a SDROI is then formed at the micropipette tip [see Fig. 3(a)].

The first frame's SDROI image is stored as a representation of the background image. Each subsequent SDROI frame is then subtracted from the background image to form a subtracted image for sperm detection. Areas of high pixel intensity in the subtracted image represent regions of movement, and areas of low pixel intensity represent regions of minimal or no change. Otsu adaptive thresholding is then applied again to the subtracted image to form a binary image [see e.g., Fig. 3(b)-(e)].

Using subtracted binary image of each frame, the algorithm searches for the position of sperm head inside the SDROI. Contour computation is performed to find every contour inside the SDROI. To minimize false detection that may be caused by contaminants/microparticles or the micropipette movement in the SDROI, an area criterion for the sperm head is used to discriminate the sperm head from other nonsperm objects. Experimentally, the sperm head has a minimum area of 40 pixels and a maximum area of 150 pixels under $20 \times$ magnification.
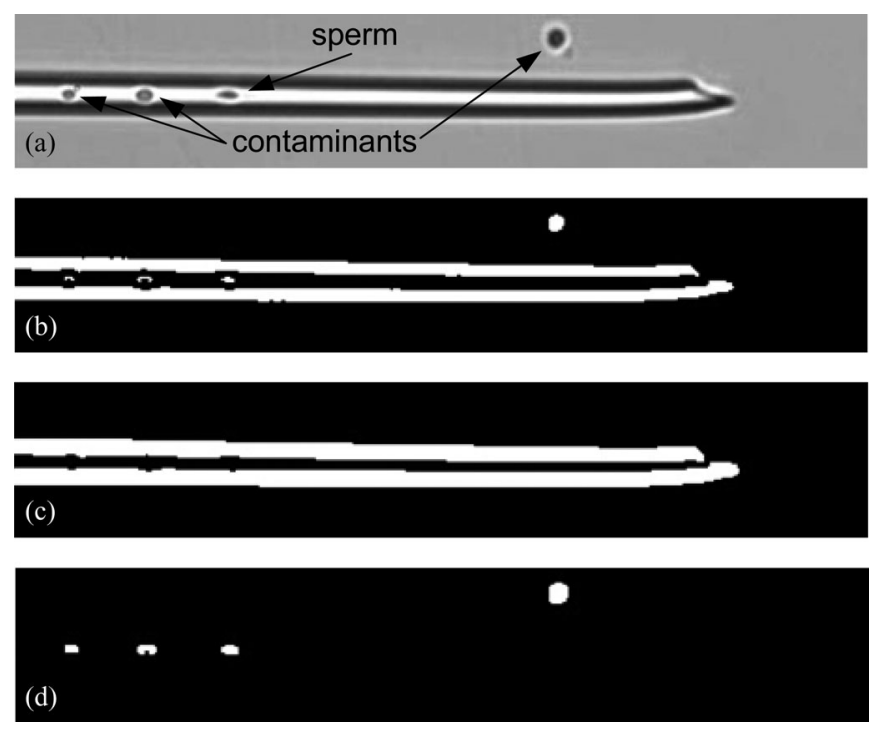

Fig. 4. Sperm tracking preprocessing steps. (a) Original image with sperm cell inside the micropipette, and contaminants on the outside of the micropipette. (b) Binary image $B_{1}(x, y)$ created through Otsu adaptive thresholding. (c) Binary image $B_{2}(x, y)$ containing only micropipette edges. (d) $B_{1}(x, y)$ after removing micropipette edges by subtracting $B_{2}(x, y)$ from $B_{1}(x, y)$.

Objects with areas outside this range were considered either the micropipette being displaced or contaminants entering the SDROI.

A virtual line drawn 20 pixels from the left of the SDROI [see Fig. 3(b)-(e)] depicts the position that the sperm must pass for detecting the sperm cell's entrance into the micropipette. When the sperm head's horizontal position is detected to be less than or equal to the virtual line position in the SDROI, the sperm head has reached the left section of the SDROI and has entered the micropipette tip [see Fig. 3(b)-(c)]. Cell detection also occurs when the sperm head position appears initially to the right of the virtual line [see Fig. 3(d)], and the sperm cell disappears from the SDROI in subsequent frames due to a high aspiration force of the micropipette. Subsequently, no contour can be found in the SDROI with a sufficient sperm head area since the sperm cell is displaced out of the SDROI and into the micropipette at a high velocity [see Fig. 3(e)].

\section{B. Sperm Cell Tracking Inside Micropipette}

After the sperm cell is aspirated into the micropipette, the sperm position obtained from visual tracking is provided to a closed-loop controller as feedback. The tracking algorithm must be robust to micropipette vibrations that can displace the micropipette and sperm cell. The tracking algorithm must also be robust to contaminants/microparticles attached to the micropipette since contaminants can occlude the sperm cell while the sperm cell is being tracked inside the micropipette.

Sperm tracking inside the micropipette is initiated by a human operator via computer mouse clicking on or within proximity to the sperm cell [see Fig. 4(a)]. The system then forms a sperm region of interest (SROI) around the clicked position. Otsu adaptive thresholding is applied to the image to form a binary image $B_{1}(x, y)$ that separates objects from the background 
[see Fig. 4(b)]. The contour of each object in the binary image is computed. The two contours with the largest areas are the micropipette's upper and lower edges.

When the sperm is inside the micropipette, it is close to the micropipette edges due to the small size of the micropipette tip. Hence, the sperm object may merge with the micropipette edges in the binary image, $B_{1}(x, y)$. This merging may cause visual tracking to fail since the tracking algorithm can take the sperm and micropipette object as one object. Thus, the micropipette edge objects are removed from $B_{1}(x, y)$ to segment the sperm object from the micropipette edge objects. To remove the micropipette edges, a binary image $B_{2}(x, y)$ containing only the micropipette edges is created by setting all other object pixels, including the sperm object in the SROI to zero [see Fig. 4(c)]. $B_{2}(x, y)$ is computed only once in the first frame, and is used to subtract the micropipette edge information from each subsequent $B_{1}(x, y)$ frame [see Fig. 4(d)].

Blob detection is performed to track the sperm in $B_{1}(x, y)$. The sperm head position $\mathbf{c}$ is determined by calculating the moment of the sperm head contour in every frame $i$. The SROI is updated accordingly in each frame to be centered at the updated sperm position. When more than one object is present in the SROI, positions of the contaminant particles found in the first frame are used to distinguish the sperm object from contaminant objects. In the first frame, the position of every object in $B_{1}(x, y)$ except the sperm is computed by finding the center of mass of contaminant objects. The minimum norm value $k$ for each object in the SROI is found with respect to the contaminant positions according to

$$
k=\min _{j \in[1, N]}\left\{\left\|\mathbf{s}-\mathbf{d}_{j}\right\|\right\}
$$

where s represents an object position inside the SROI, and $\mathbf{d}_{j}$ represents the position of contaminant object $j$. Contaminant objects inside the SROI all have $k$ values of 0 or close to 0 , since these objects have positions that match those found in $\mathbf{d}$. Thus, the object in the SROI that produces the largest $k$ with respect to the contaminant positions is considered the sperm object. When the sperm is occluded by a contaminant, the algorithm tracks the sperm and contaminant as one object until the sperm moves away from the contaminant (see Fig. 5).

When no objects are present within the SROI, the direction vector of the sperm is used to predict the position of the sperm in the next frame. The scenario of no objects present in the SROI may occur in cases where the sperm is being moved with a large velocity. Consequently, the sperm may appear blurry in certain frames, resulting in the sperm being absent from the binary image. The direction vector $\overline{\mathbf{v}}(i)$ of the sperm is calculated as

$$
\overline{\mathbf{v}}(i)=\frac{1}{30} \sum_{k=0}^{29}(\mathbf{c}(i-k)-\mathbf{c}(i-k-1)) .
$$

Accordingly, the SROI moves in the direction predicted by the direction vector. In the updated SROI position, the same blob detection algorithm is used to find the sperm being tracked. If the sperm cannot be found after a certain number of frames, sperm tracking is stopped, and the system considers the sperm lost.
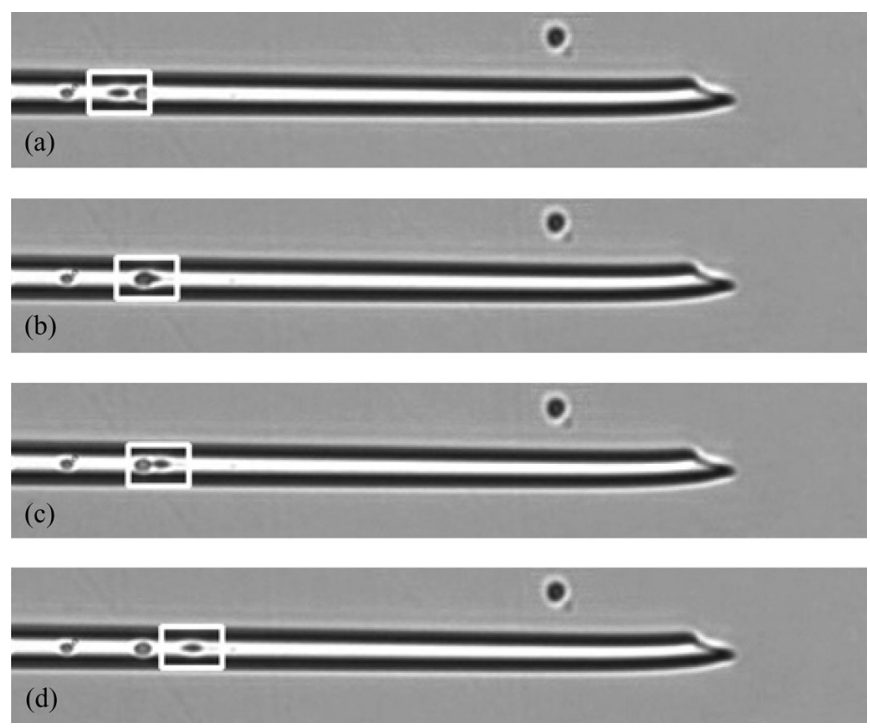

Fig. 5. Occlusion tracking. (a) Sperm is moving to the right. The SROI (shown by the white box) contains both the sperm and a contaminant. (b) Sperm continues to move to the right and is occluded by the contaminant. (c) Sperm and contaminant are both inside the SROI. Tracking continues on sperm. (d) Sperm moves away from the contaminant and is tracked in the SROI.

There were instances where the micropipette vibrates or displaces from its original position although the cell manipulation system was on top of an antivibration table. Micropipette vibration or displacements can be caused by disturbances in the medium, or movement of the platform on which the microscope is situated. When the micropipette is displaced, the tracking algorithm can fail if $B_{2}(x, y)$ and the contaminant positions are not recomputed. Thus, an algorithm was developed to detect micropipette displacement. The detection algorithm triggers the system to pause tracking and update $B_{2}(x, y)$ and contaminant positions.

For each frame, the top left positions of the lower and upper micropipette edges are calculated by fitting a rectangle to each micropipette edge. The top left position of each rectangle is stored as the top left position of each micropipette edge for each frame. When the Euclidean norm between the top left position in the current frame and the previous frame surpasses a threshold value (e.g., 1 pixel), micropipette vibration/displacement is detected, and tracking is paused for 15 frames. After 15 frames, the system computes the new contaminant positions and $B_{2}(x, y)$. Tracking is then resumed, and the micropipette displacement distance is added to the last successfully computed SROI to provide the system with the updated initial SROI position.

\section{Dynamics of Cell Motion}

For controlling the sperm cell's position inside the micropipette, the dynamics of cell motion must be integrated in the closed-loop position controller. Cell positioning is schematically shown in Fig. 6. Major components include a syringe, a micropipette, and a connecting tube. The syringe and connecting tube are filled with mineral oil. The inner space of micropipette typically consists of three segments: mineral oil, air, and culture 


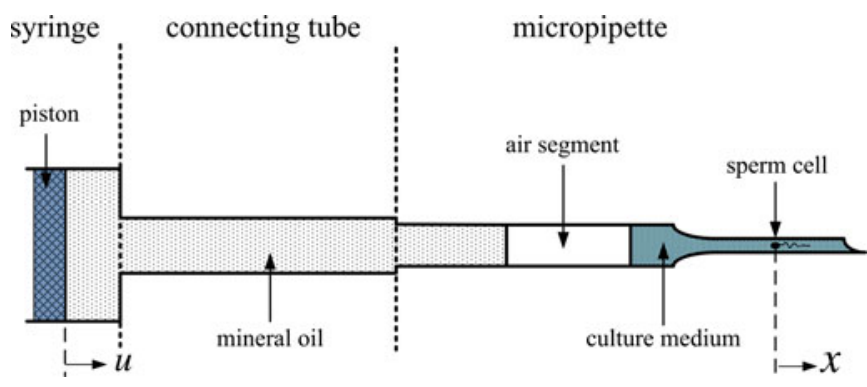

Fig. 6. Schematic diagram of sperm moving inside a micropipette linked to a syringe.

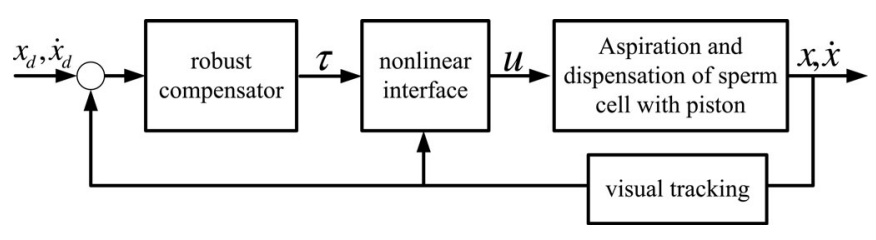

Fig. 7. Block diagram of the control system.

medium. A sperm cell moves together with the culture medium segment (no relative motion within the culture medium).

Motion of the syringe piston is denoted by $u$, and the position of the sperm cell is denoted by $x$. The initial volume of the air segment inside the micropipette is $V_{0}$. The cross-sectional area of the air segment is $A$. The initial pressure is $P_{0}$ that is equal to the atmospheric pressure $P_{\text {atm }}$ at equilibrium. During aspiration and dispensing, the volume of the air segment inside the micropipette changes as

$$
V=x A_{1}-u A_{2}+V_{0}=\left(\varepsilon_{1} x-\varepsilon_{2} u\right) A+V_{0}
$$

where $\varepsilon_{1}$ is the ratio of the cross-sectional area of the micropipette tip $A_{1}$ to the air segment $A$; and $\varepsilon_{2}$ is the ratio of the cross-sectional area of the piston $A_{2}$ to the air segment $A$.

To calculate the pressure of the air segment, Boyle's law gives $P V=P_{0} V_{0}$. Applying Newton's law, the dynamic equations of the moving culture medium containing a sperm cell is

$$
m \ddot{x}+c \dot{x}=\frac{A V_{0} P_{0}}{\left(\varepsilon_{1} x-\varepsilon_{2} u\right) A+V_{0}}-A P_{0}
$$

where $m$ is the mass of the culture medium containing the sperm cell, and $c$ is the damping of the culture medium.

\section{Controller Design}

In the dynamic system expressed in (4), the control input is the piston's motion $u$. System output is the motion of the sperm cell $x$. The system is nonlinear, and the mass $m(x)$ changes with time during cell aspiration and dispensing, and the damping coefficient varies with the viscosity of medium. Hence, we linearized the system and designed a robust controller for the linearized system that has uncertain mass and varying damping. Fig. 7 shows the block diagram of the control system.

\section{A. Input Linearization}

To linearize the control input, the right-hand side of (4) is denoted as

$$
\tau=k_{d}\left(\dot{x_{d}}-\dot{x}\right)+k_{p}\left(x_{d}-x\right)
$$

where $k_{d}$ is the derivative gain, $k_{p}$ is the proportional gain, and $x_{d}$ is the target sperm cell position.

Combing (5) with (3), the control input to the syringe is

$$
u=\frac{\varepsilon_{1}}{\varepsilon_{2}}\left(\frac{-P_{0} V_{0}}{k_{d}\left(\dot{x_{d}}-\dot{x}\right)+k_{p}\left(x_{d}-x\right)+A P_{0}}+\frac{V_{0}}{A}+x\right)
$$

where control gains $k_{p}$ and $k_{d}$ must be determined for optimal control performance.

\section{B. Controller}

In dynamic equation (4), the mass $m$ of culture medium that contains the sperm cell changes during cell positioning. $m$ increases when culture medium is aspirated into the micropipette, and decreases when it is dispensed out of the micropipette. Additionally, system damping, $c$ varies with culture medium viscosity. With considerations of mass and damping variation, robust control was applied to determine $k_{p}$ and $k_{d}$.

The state-space presentation of (4) is

$$
\left(\begin{array}{l}
\dot{y}_{1} \\
\dot{y}_{2}
\end{array}\right)=\left(\begin{array}{cc}
0 & 1 \\
0 & -\frac{c}{m}
\end{array}\right)\left(\begin{array}{l}
y_{1} \\
y_{2}
\end{array}\right)+\left(\begin{array}{c}
0 \\
\frac{1}{m}
\end{array}\right) \tau
$$

and can be simplified into

$$
\dot{X}=A X+B^{*} \tau
$$

where

$$
X=\left(\begin{array}{l}
y_{1} \\
y_{2}
\end{array}\right), A=\left(\begin{array}{cc}
0 & 1 \\
0 & -\frac{c}{m}
\end{array}\right), B^{*}=\left(\begin{array}{c}
0 \\
\frac{1}{m}
\end{array}\right)
$$

$y_{1}=x$, and $y_{2}=\dot{x}$. The robust control strategy presented shortly was implemented by translating a robust control problem into an optimal control problem [25].

1) Define Robust Control Problem: Defining $-c / m$ as uncertainty variable $p$, and defining $-1 / m$ as uncertainty $q$, we can rewrite the state-space equation (8) with uncertainty as

$$
\dot{X}=A(p) X+B D(q) \tau
$$

where

$$
A(p)=\left(\begin{array}{ll}
0 & 1 \\
0 & p
\end{array}\right), \quad B=\left(\begin{array}{l}
0 \\
1
\end{array}\right), \quad D(q)=q .
$$

To describe the robust control problem, three assumptions were made. 1) There exists a nominal value $p_{0}$ of $p$ $\left(p_{\min }<p_{0}<p_{\max }<0\right)$ such that the controllability matrix $C=\left(B, A\left(p_{0}\right) B\right)$ is of full rank. 2) There exists a constant $D=q_{0}$ such that $0<q_{0} \leq q$. 3) $A(p)$ is bounded. Based on these assumptions, the closed-loop system dynamics is rewritten as

$$
\dot{X}=A(p) X+B D(\tau+E(q) \tau)
$$

where $E(q)=D^{-1} D(q)-I \geq 0$. The robust control design problem becomes the determination of a feedback control law, 
$\tau=K X$ such that the closed-loop system $\dot{X}=A(p) X+$ $B D(K X+E(q) K X)$ is asymptotically stable for all $p$ and $q$.

In order to solve the robust control problem, the uncertainty $A(p)-A\left(p_{0}\right)$ must be decomposed into the sum of a matched component and an unmatched component by projecting it onto the range of $B D$, namely

$$
\begin{aligned}
A(p)-A\left(p_{0}\right)= & (B D)(B D)^{+}\left(A(p)-A\left(p_{0}\right)\right. \\
& +\left(I-(B D)(B D)^{+}\right)\left(A(p)-A\left(p_{0}\right) .\right.
\end{aligned}
$$

Two upper bounds on the uncertainty are defined to translate the robust control problem into an optimal control problem, as

$$
\begin{array}{r}
\left(A(p)-A\left(p_{0}\right)\right)^{T}(B D)^{+^{T}}(B D)^{+}\left(A(p)-A\left(p_{0}\right)\right) \leq G \\
\alpha^{-2}\left(A(p)-A\left(p_{0}\right)\right)^{T}\left(\left(A(p)-A\left(p_{0}\right)\right) \leq H\right.
\end{array}
$$

where $\alpha \geq 0$ is a design parameter, $(\bullet)^{+}$is pseudoinverse of a matrix, and $(\bullet)^{T}$ is transpose of a matrix.

2) Define Optimal Control Problem: An auxiliary system is built as

$$
\dot{X}=A\left(p_{0}\right) X+(B D) \tau+\alpha\left(I-(B D)(B D)^{+}\right) \eta .
$$

The robust controller problem is then translated into an optimal problem, namely, finding a feedback control law $\tau=K X$ and $\eta=M X$ that minimize the cost functional (LQR problem)

$$
\int\left(X^{T}\left(G+\beta^{2} H+\gamma^{2} I\right) X+\tau^{T} \tau+\beta^{2} \eta^{T} \eta\right) d t
$$

where $\beta \geq 0$ and $\gamma \geq 0$ are design parameters. Since $\left(A\left(p_{0}\right), B\right)$ is stabilizable (the controllability matrix $C=\left(B, A\left(p_{0}\right) B\right)$ is of full rank), $G \geq 0$, and $H \geq 0$, the solution to the $\mathrm{LQR}$ problem exists. Based on the LQR theory, the control law is

$$
\left(\begin{array}{l}
\tau \\
\eta
\end{array}\right)=\left(\begin{array}{c}
-(B D)^{T} S X \\
-\alpha \beta^{-2}\left(I-(B D)(B D)^{+}\right) S X
\end{array}\right) .
$$

The matrix $S$ is solved from the Riccati equation

$$
\begin{aligned}
& S A\left(p_{0}\right)+A\left(p_{0}\right)^{T} S+G+\beta^{2} H+\gamma^{2} I-S\left((B D)(B D)^{T}\right. \\
& \left.\quad+\alpha^{2} \beta^{-2}\left(I-(B D)(B D)^{+}\right)^{2}\right) S=0 .
\end{aligned}
$$

3) Proof and Solution: As detailed in Appendix A, the solution $\tau=K X$ and $\eta=M X$ to the $\mathrm{LQR}$ problem is a solution to the robust control problem. Therefore, the robust controller design task is to choose $\alpha, \beta$, and $\rho$ such that the solution $\tau=K X$ and $\eta=M X$ to the LQR problem satisfies $\beta^{2} I-2 \rho^{2} M^{T} M>$ 0 . Then, $\tau=K X=-(B D)^{T} S X$ is a solution to the robust control problem. Note that $\beta^{2} I-2 \rho^{2} M^{T} M>0$ is a sufficient but not necessary condition. Substituting $K=\left(k_{p}, k_{d}\right)$ into (6), the system control input $u$ (i.e., the position of syringe piston) is obtained.

\section{EXPERIMENT RESULTS AND DISCUSSION}

Experiments of sperm cell detection and tracking were first conducted to validate the computer vision algorithms. The algorithm was then integrated into the robust controller to form a
TABLE I

SPERM TRACKING RESULTS

\begin{tabular}{lll}
\hline velocity $(\mu \mathrm{m} / \mathrm{sec})$ & success & failure \\
\hline $0<\bar{u}<35$ & 26 & 0 \\
$35 \leq \bar{u}<70$ & 23 & 1 \\
$70 \leq \bar{u} \leq 100$ & 48 & 2 \\
\hline
\end{tabular}

complete closed-loop control system. Experiments of positioning a sperm cell to a target position were performed using the robust controller, and the performance was compared to that of a standard proportional derivative (PD) controller.

Besides the hundreds of sperm cells used in system development, the system tested 100 sperm cells to quantify the sperm aspiration detection success rate. Sperm aspiration detection success rate is defined as the percentage of true positive detection cases in which the sperm cell is aspirated into the micropipette. The algorithm successfully detected 98 out of 100 trials (i.e., $98 \%$ success rate). We observed that false positive detection can occur when other sperm cell swim into the SDROI. In this failure case, the algorithm falsely detected the sperm as passing the virtual line.

Additionally, 100 trials of sperm tracking inside the micropipette were performed to evaluate sperm tracking success rate. Tracking success rate is defined as the ability of the tracking algorithm to follow the sperm inside the micropipette as the sperm is moved from the left most side of the micropipette to the micropipette tip. The tracking algorithm is evaluated independently from the closed-loop controller. The dispensing pressure was controlled manually by a human operator for the 100 sperm tracking trials. Sperm cells were moved at a range of different velocities to evaluate the robustness of the tracking algorithm.

The sperm's velocity $\bar{u}$ is calculated by converting the direction vector $\bar{v}$ from pixels $/ \mathrm{s}$ to $\mu \mathrm{m} / \mathrm{s}$. Results are categorized into three groups, based on sperm velocity: $0 \mu \mathrm{m} / \mathrm{s}<\bar{u}<35 \mu \mathrm{m} / \mathrm{s}$, $35 \mu \mathrm{m} / \mathrm{s} \leq \bar{u}<70 \mu \mathrm{m} / \mathrm{s}$, and $70 \mu \mathrm{m} / \mathrm{s} \leq \bar{u} \leq 100 \mu \mathrm{m} / \mathrm{s}$. The tracking algorithm is capable of tracking sperm velocities above $100 \mu \mathrm{m} / \mathrm{s}$. However, there were instances when the algorithm lost track of the sperm due to the sperm head's low contrast and blurred appearance at velocities greater than $100 \mu \mathrm{m} / \mathrm{s}$. Hence, the upper threshold of the sperm's velocity was set in the system at $100 \mu \mathrm{m} / \mathrm{s}$. Overall, the system achieved a sperm tracking success rate of $97 \%$. Details are summarized in Table I.

As summarized in Table I, three tracking failures occurred. One failure case was when the sperm became out of focus in the micropipette, the sperm head's appearance inside the micropipette becomes blurry and blends into the background. Thus, in the out-of-focus case, the tracking algorithm tends to lose track of the sperm. Another failure case happened when the sperm adhered to the micropipette in areas of the micropipette with higher adhesiveness. Higher dispensing pressure was required to move the sperm in this case. When the sperm progressed toward an area of the micropipette with lower adhesiveness, the sperm velocity increased to a value larger than 100 
$\mu \mathrm{m} / \mathrm{s}$. At this velocity, the tracking algorithm lost track of the sperm.

The last failure case was when a sperm cell outside the micropipette moved into the SROI in which the sperm of interest inside the micropipette is being tracked, the outside sperm merged with the sperm of interest in the preprocessed binary image. The outside sperm moved in the same direction as the sperm of interest, and hence, the two sperm direction vectors were the same. After the two sperm cells separated, the algorithm incorrectly tracked the sperm outside the micropipette. This failure case occurred only once, and hence, is considered a rare case. For the majority of cases where outside sperm cells enter the region of interest, the direction vector and the vertical restriction of the sperm to the vertical position of the micropipette enable the algorithm to effectively distinguish the sperm of interest from outside sperm cells that happen to enter the SROI.

The sperm tracking information was provided in real time to the closed-loop controller for positioning the sperm cell to a target location inside the micropipette. The diameters of the cross section of the syringe piston, air segment, and the micropipette tip are $3.26 \mathrm{~mm}, 750 \mu \mathrm{m}$, and $6 \mu \mathrm{m}$, respectively. The initial length of the air segment inside the micropipette is $15 \mathrm{~mm}$. Based on the controller design methodology [see (16)], the robust controller was designed as

$$
\begin{aligned}
u= & 3.3874 \\
& \times 10^{-6}\left(\frac{-4.4179 \times 10^{-4}}{k_{d}\left(\dot{x_{d}}-\dot{x}\right)+k_{p}\left(x_{d}-x\right)+4.4179 \times 10^{-2}}\right. \\
& +0.01+x)
\end{aligned}
$$

where $k_{p}=1.4$, and $k_{d}=1671.2$.

To validate the control performance of the robust controller, the controller was applied to move a sperm cell from an initial position to a target position inside the micropipette, as shown in Fig. 8(a). The distance between the target position to the micropipette tip was set to be slightly larger than one sperm length. Hence, when the sperm head was moved to the target position, the sperm cell's tail was close to the opening of the micropipette tip. The distance between the original position of the sperm cell and the target position, shown in Fig. 8 was 420 pixels (i.e., $200 \mu \mathrm{m}$ ).

Of the 100 sperm cells tested in the experiments, the visually servoed robust controller was capable of moving a sperm cell to the target position quickly and stably. The average settling time was $8 \mathrm{~s}$ and the steady-state error was zero. There were a few cases where a small overshoot was observed [see Fig. 8(b)]. However, the largest overshoot was less than 3 pixels. The success rate for positioning sperm cells was $100 \%$ in all cases where sperm tracking was successful.

A standard PD controller was also implemented under the same experimental conditions for performance comparison with the robust controller. The PD control gains were optimized based on extensive trial and error to achieve performance comparable to that of the robust controller (e.g., small overshoot and short settling time). The optimized PD controller required a long settling time (e.g., $10 \mathrm{~s}$ ) when a large derivative gain was used. As
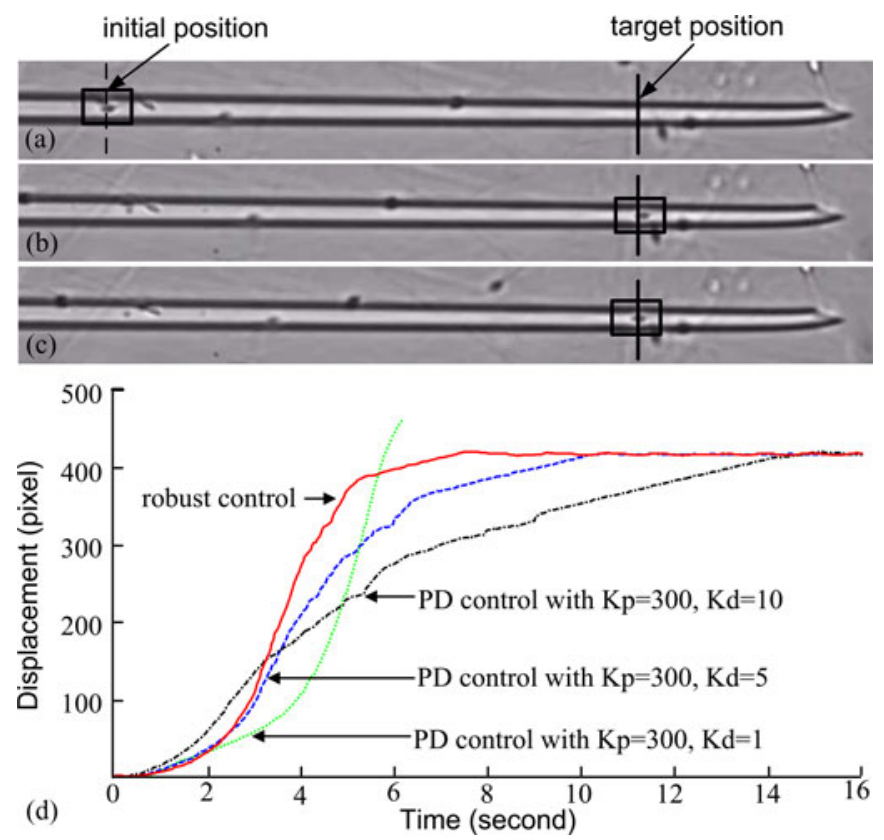

Fig. 8. Positioning a sperm cell inside the micropipette with a robust controller. (a) Sperm cell at initial position. (b) Slight overshoot. (c) Sperm cell reached target position with zero steady-state error. (d) Performance comparison between robust controller and PD controller.

the derivative gain was reduced, the overshoot of the PD controller increased dramatically. As shown in Fig. 8(d), the sperm cell was dispensed completely out of the micropipette when the derivative gain was set to be 1 due to the large overshoot. A larger derivative gain (e.g., 5 and 10) was effective in positioning a sperm cell; however, the robust controller significantly outperforms the PD controller in terms of efficiency, overshoot, and accuracy. The robustness to variations in control parameter values also proved its suitability for single-cell position control.

\section{CONCLUSION}

This paper presented single-cell manipulation techniques for automated aspiration of a cell into a micropipette and positioning of the cell inside the micropipette. Computer vision algorithms were described for visually detecting and tracking a cell outside and inside the micropipette. High success rates of $98 \%$ for cell detection and $97 \%$ for cell tracking were obtained. A nonlinear dynamic model of cell motion inside the micropipette was established. Based on the dynamic model and visual tracking, a closed-loop robust controller was designed against mass and damping variation. Experimental results demonstrated that the robust controller is stable and has short settling time and zero steady-state error, outperforming standard PD control. The automated system is capable of aspirating a single cell into a micropipette within $2 \mathrm{~s}$ (versus typically $10 \mathrm{~s}$ by highly skilled operators) and accurately positioning the cell to a target position inside the micropipette within $8 \mathrm{~s}$ (versus typically $25 \mathrm{~s}$ by highly skilled operators). 


\section{APPENDIX A}

To prove that the solution $\tau=K X$ and $\eta=M X$ to the LQR problem is a solution to the robust control problem, we define a Lyapunov function for the robust design problem as the minimum cost of the optimal control of the auxiliary system. The Lyapunov function is

$$
\begin{gathered}
V(x)=\min _{\tau, \eta} \int_{0}^{\infty}\left(X^{T}\left(G+\beta^{2} H+\gamma^{2} I\right) X\right. \\
\left.+\tau^{T} \tau+\beta^{2} \eta^{T} \eta\right) d t .
\end{gathered}
$$

When $x \neq 0, V(X)>0$; and when $x=0, V(X)=0$. Based on the definition, $V(x)$ must satisfy the Hamilton-Jacobi-Bellman equation and is reduced to

$$
\begin{aligned}
& \min _{\tau, \eta}\left(X^{T}\left(G+\beta^{2} H+\gamma^{2} I\right) X+\tau^{T} \tau+\beta^{2} \eta^{T} \eta\right. \\
& \left.\quad+V_{X}^{T}\left(A\left(p_{0}\right) X+B D \tau+\alpha\left(I-(B D)(B D)^{+}\right) \eta\right)\right) .
\end{aligned}
$$

Based on the optimal control solution $\tau=K X$ and $\eta=$ $M X,(20)$ is divided into

$$
\begin{aligned}
& \left(X^{T}\left(G+\beta^{2} H+\gamma^{2} I\right) X+X^{T} K^{T} K X\right. \\
& \quad+\beta^{2} X^{T} M^{T} M X+V_{X}^{T}\left(A\left(p_{0}\right) X\right. \\
& \left.\quad+B D K X+\alpha\left(I-(B D)(B D)^{+}\right) M X\right)=0 \\
& 2 X^{T} K^{T}+V_{X}^{T} B D=0 \\
& 2 \beta^{2} X^{T} M^{T}+V_{X}^{T} \alpha\left(I-(B D)(B D)^{+}\right)=0
\end{aligned}
$$

with (10), the derivative of the Lyapunov function can be written as

$$
\begin{aligned}
V(X) & =V(X)^{T} \dot{X} \\
& =V_{X}^{T}(A(p) X+B D(K X+E(p) K X) .
\end{aligned}
$$

Equation (24) can be rewritten as

$$
\begin{aligned}
V \dot{(X)}= & V_{X}^{T}\left(A\left(p_{0}\right) X+B D K X\right. \\
& \left.+\alpha\left(I-(B D)(B D)^{+}\right) M X\right) \\
& \left.+V_{X}^{T}(B D)(B D)^{+}\right)\left(A(p)-A\left(p_{0}\right)\right) X \\
& +V_{X}^{T}\left(I-(B D)(B D)^{+}\right)\left(A(p)-A\left(p_{0}\right)\right) X \\
& -V_{X}^{T} \alpha\left(I-(B D)(B D)^{+}\right) M X \\
& +V_{X}^{T} B D E(p) K X .
\end{aligned}
$$

Combining (21), (22), and (23), (25) can be rewritten as

$$
\begin{aligned}
V(X)= & -X^{T}\left(G+\beta^{2} H+\gamma^{2} I\right) X-X^{T} K^{T} K X \\
& \left.-\rho^{2} X^{T} M^{T} M X-2 X^{T} K^{T}(B D)^{+}\right)(A(p) \\
& \left.-A\left(p_{0}\right)\right) X-2 \alpha^{-1} \rho^{2} X^{T} M^{T}\left(A(p)-A\left(p_{0}\right)\right) X \\
& +2 \rho^{2} X^{T} M^{T} M X-2 X^{T} K^{T} E(p) K X .
\end{aligned}
$$

With (12) and (13), (26) becomes

$$
\begin{aligned}
V(X) \leq & -X^{T}\left(\beta^{2} I-2 \rho^{2} M^{T} M\right) X \\
& -2 X^{T} K^{T} E(p) K X
\end{aligned}
$$

since $E(q)=D^{-1} D(q)-I \geq 0,(27)$ becomes

$$
V(X) \leq-X^{T}\left(\beta^{2} I-2 \rho^{2} M^{T} M\right) X .
$$

With the condition $\beta^{2} I-2 \rho^{2} M^{T} M>0$, we can conclude that $V(X)<0$ when $X \neq 0, V(X)=0$ when $X=0$. According to the Lypayunov stability theorem, the robust control system is stable for all uncertainty $p$ and $q$, namely, $\tau=K X$ is a solution to the robust control problem.

\section{REFERENCES}

[1] C. Sean, E. F. Simonds, P. Qiu, el-AD Amir, P. O. Krutzik, R. Finck, R. V. Bruggner, R. Melamed, A. Trejo, O. I. Ornatsky, R. S. Balderas, S. K. Plevritis, K. Sachs, D. Pe'er, S. D. Tanner, and G. P. Nolan, "Single cell mass cytometry of differential immune and drug responses across a human hematopoietic continuum," Science, vol. 332, pp. 687-696, 2011.

[2] R. S. John, S. Ghaemmaghami, J. Ihmels, D. K. Breslow, M. Noble, J. L. DeRisi, and J. S. Weissman, "Single cell proteomic analysis of $S$. cerevisiae reveals the architecture of biological noise," Nature, vol. 441, pp. 840-846, 2006.

[3] H. Kortmann, L. M. Blank, and A. Schmid, "Single cell analysis reveals unexpected growth phenotype of S. cerevisiae," Cytometry A, vol. 75 pp. 130-139, 2009.

[4] J. S. Schneider, J. M. Vitale, A. Terzic, and D. Fraidenraich, "Blastocyst injection of embryonic stem cells: A simple approach to unveil mechanisms of corrections in mouse models of human disease," Stem Cell Rev. Rep., vol. 5, pp. 369-377, 2009.

[5] A. Kuliev and S. Rechitsky, "Polar body-based preimplantation genetic diagnosis for Mendelian disorders," Mol. Human Reproduct., vol. 17, pp. 275-285, 2011.

[6] C. Yi, S. Ji, and M. Yang, "Microfluidics technology for manipulation and analysis of biological cells," Analytica Chimica Acta, vol. 560, pp. 1-23, 2006.

[7] K. Ino, M. Okochi, N. Konishi, M. Nakatochi, R. Imai, M. Shikida, A. Ito, and H. Honda, "Cell culture arrays using magnetic force-based cell patterning for dynamic single cell analysis," Lab Chip, vol. 8, pp. 134-142, 2008.

[8] X. Wang, S. Chen, M. Kong, Z. Wang, K. Costa, R. Li, and D. Sun, "Enhanced cell sorting and manipulation with combined optical tweezer and microfluidic chip technologies," Lab Chip, vol. 11, pp. 3656-3662, 2011.

[9] A. Docoslis, N. Kalogerakis, and L. A. Behie, "Dielectrophoretic forces can be safely used to retain viable cells in perfusion cultures of animal cells," Cytotechnology, vol. 30, pp. 133-142, 1999.

[10] L. Gherardini, C. M. Cousins, J. J. Hawkes, J. Spengler, S. Radel, H. Lawler, B. Devcic-Kuhar, and M. Groschl, "A new immobilisation method to arrange particles in a gel matrix by ultrasound standing waves," Ultrasound Med. Biol., vol. 31, pp. 261-272, 2005.

[11] U. Demirci and G. Montesano, "Cell encapsulating droplet vitrification," Lab Chip, vol. 7, pp. 1428-1433, 2007.

[12] R. M. Hochmuth, "Micropipette aspiration of living cells," J. Biomech., vol. 33, pp. 15-22, 2000.

[13] W. H. Wang, X. Y. Liu, and Y. Sun, "High-throughput automated injection of individual biological cells," IEEE Trans. Autom. Sci. Eng., vol. 6, no. 2, pp. 209-219, Apr. 2009.

[14] K. Elder and B. Dale, In-Vitro Fertilization, 3rd ed. Cambridge, U.K.: Cambridge Univ. Press, 2011.

[15] Z. Lu, C. Moraes, G. Ye, C. A. Simmons, and Y. Sun, "Single cell deposition and patterning with a robotic system," PLoS One, vol. 5, p. e13542, 2010.

[16] H. A. Yasser, R. H. Mark, and R. M. Meldrum, "Automated selection and placement of single cells using vision-based feedback control," IEEE Trans. Autom. Sci. Eng., vol. 7, no. 3, pp. 598-606, Jul. 2010.

[17] R. J. Sherins, L. P. Thorsell, A. Dorfmann, L. Dennison-Lagos, L. P. Calvo, L. Krysa, C. B. Coulam, and J. D. Schulman, "Intracytoplasmic sperm injection facilitates pregnancies even in the most severe forms of male infertility," Fertility Sterility, vol. 64, pp. 369-375, 1995.

[18] Z. Lu, X. P. Zhang, C. Leung, N. Esfandiari, R. F. Casper, and Y. Sun, "Robotic ICSI (Intracytoplasmic sperm injection)," IEEE Trans. Biomed. Eng., vol. 58, no. 7, pp. 2102-2108, Jul. 2011.

[19] M. A. Alyassin, S. Moon, H. O. Keles, F. Manzur, R. L. Lin, E. Haeggstrom, D. R. Kurtzkes, and U. Demirci, "Rapid automated cell 
quantification on HIV microfluidic devices," Lab Chip, vol. 9, pp. 3364$3369,2009$.

[20] S. Huh, D. F. Ker, R. Bise, M. Chen, and T. Kanade, "Automated mitosis detection of stem cell populations in phase-contrast microscopy images," IEEE Trans. Med. Imag., vol. 30, no. 3, pp. 586-596, Mar. 2011.

[21] X. Chen, X. Zhou, and S. T. C. Wong, "Automated segmentation, classification and tracking of cancer cell nuclei in time-lapse microscopy," IEEE Trans. Biomed. Eng., vol. 53, no. 4, pp. 762-766, Apr. 2006.

[22] X. Zhou, F. Li, J. Yan, and S. T. C. Wong, "A novel cell segmentation method and cell phase identification using Markov model," IEEE Trans. Inf. Technol. Biomed., vol. 13, no. 2, pp. 152-157, Mar. 2009.

[23] W. H. Wang, X. Y. Liu, and Y. Sun, "High-throughput automated injection of individual biological cells," IEEE Trans. Autom. Sci. Eng., vol. 6, no. 2, pp. 209-219, Apr. 2009.

[24] C. Leung, Z. Lu, N. Esfandiari, R. F. Casper, and Y. Sun, "Automated sperm immobilization for intracytoplasmic sperm injection," IEEE Trans. Biomed. Eng., vol. 58, no. 4, pp. 935-942, Apr. 2011.

[25] F. Lin, Robust Control Design: An Optimal Control Method. Chichester, U.K.: Oxford Univ., 2007.

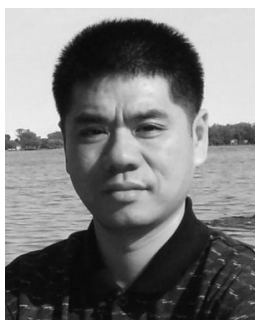

XuPing Zhang (M'11) received the B.Eng. degree in mechanical engineering from Chongqing University, Chongqing, China, in 1992, and the Ph.D. degree in mechanical engineering from the University of Toronto, Toronto, ON, Canada, in 2009. He is currently a Postdoctoral Fellow in the Advanced Micro and Nanosystems Laboratory, Mechanical and Industrial Engineering Department, University of Toronto, Toronto, ON, Canada. His research interests include robotics, dynamics and control, vibration theory, and mechanisms.

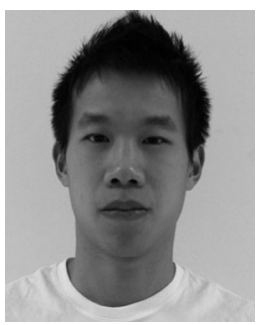

Clement Leung is a Master student in the Electrical and Computer Engineering Department at the University of Toronto. He received his B.A.Sc. in Electrical Engineering from the University of British Columbia in 2009.

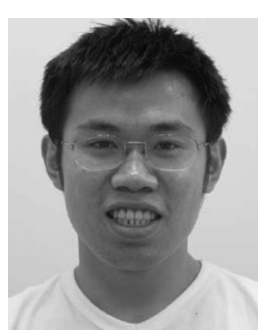

Zhe Lu (S'07-M'10) received the B.Eng. degree in control engineering from Harbin Institute of Technology, Harbin, China, in 2002, and the Ph.D. degree in mechanical engineering from the National University of Singapore, Singapore, in 2007. He is currently a Postdoctoral Fellow in the Advanced Micro and Nanosystems Laboratory, Mechanical and Industrial Engineering Department, University of Toronto, Toronto, ON, Canada. His research interests include robotics, automation, and mechatronics.

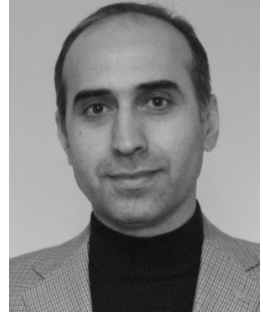

Navid Esfandiari completed his residency training and Ph.D. in Medical Immunology at Tehran University School of Medicine in 1994. He was an Assistant Professor of Immunology in Tehran, from 1994 to 2000. His fellowship was in Andrology and infertility from the Cleveland Clinic, Cleveland, Ohio from 2000 to 2002 . He is a board certified HCLD by the American Board of Bioanalysis. Dr Esfandiari is Director of the ART Laboratories at TCART, and adjunct Professor in the Department of Obstetrics and versity of Tehran, Iran Gynecology at the University of Toronto, and Uni-

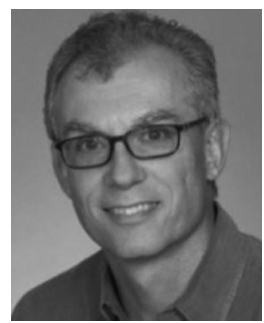

University of Toronto.
Robert F. Casper is the Camille Dan Family Research Chair in Translational Cell Biology and a Senior Investigator in the Samuel Lunenfeld Research Institute at Mount Sinai Hospital. Dr Casper is also Professor of the Division of Reproductive Endocrinology and Infertility in the Department of Obstetrics and Gynecology at the University of Toronto, with cross appointments in the Departments of Physiology, Medicine (Division of Endocrinology), The Institute of Medical Sciences, and in the Postgraduate Department of the Faculty of Dentistry at the

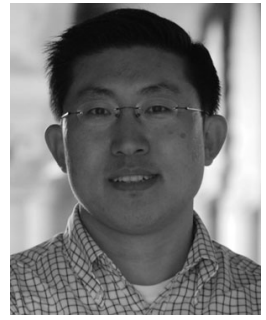

Yu Sun (S'01-M'03-SM'07) is an associate professor at the University of Toronto. He received his Ph.D. degree in mechanical engineering from the University of Minnesota, Minneapolis, in 2003. Before joining the faculty of Toronto in July 2004, he was a research scientist at the Swiss Federal Institute of Technology (ETH-Zurich). His research includes design and fabrication of MEMS/NEMS devices; micronanorobotic manipulation under optical and electron microscopes; and manipulation and characterization of biological cells, biomolecules, and nanomaterials using robotics and MEMS/NEMS/microfluidics approaches. 